\title{
Velocity Gradient Optimization in a Perforated Tray- Type Flocculator using OpenFOAM: CFD as a Tool in Water Treatment
}

\author{
P. A. Melo ${ }^{1 \dagger}$, E. R. C. G. Freire ${ }^{2}$, J. L. Ansoni ${ }^{2}$, L. F. C. Oliveira ${ }^{1}$ and C. S. Franco ${ }^{1}$ \\ ${ }^{1}$ Department of Water Resources and Sanitation, Lavras Federal University, Postal Code 3037, \\ Campus Universitario, Lavras, Brazil \\ ${ }^{2}$ Department of Mathematics and Applied Mathematics, Lavras Federal University, Postal Code \\ 3037, Campus Universitario, Lavras, Brazil \\ ${ }^{\dagger}$ Corresponding Author Email: pamela.ap.melo@gmail.com
}

(Received November 14, 2020; accepted September 20, 2021)

\begin{abstract}
Analysis of the velocity gradient of flocculators through Computational Fluid Dynamics (CFD) simulation can be essential to the optimization of hydraulic conditions in Water Treatment Plants. This study aims to simulate the velocity field in the last tank of a perforated tray-type flocculator and quantify locally velocity gradient $(\mathrm{G})$ through CFD. This stage of flocculation has a higher risk of flocs rupture when there are not adequate conditions. Thus, simulations occurred at the flocculator current operational flow rate $\left(7 \mathrm{l} \mathrm{s}^{-1}\right)$, and at full capacity $\left(91 \mathrm{~s}^{-1}\right)$. An alternative cost-effective and easy to implement modification was tested by increasing the number of orifices in the flocculator trays. As result, the velocity field indicates the formation of dead zones at the edges of the tank for all simulations, which facilitates short circuit occurrences. This is an indicator of reductions in water treatment efficiency. After structural modifications, simulations indicate a reduction in dead zone areas. Plus, as the flow rate increases, the maximum $G$ inside the structure increases considerably (184 to $266 \mathrm{~s}^{-1}$ ), causing a risk for floc rupture. However, changing the number of orifices from 22 to 33 creates conditions for the flocculator to operate at higher flow rates without increasing the velocity gradient.
\end{abstract}

Keywords: Water treatment plant; Turbulence; Floc rupture; Orifices; Hydraulics.

\section{INTRODUCTION}

Flocculation is an important step in either drinking water and some wastewater treatment plants. This process is preceded by a coagulation phase, which consists of the application of chemicals such as Ferric Chloride, whose purpose is to destabilize colloidal particles charges. Therefore, it becomes possible for these particles to aggregate into larger diameters. Flocculation is stimulated by fluid agitation to form flocs, which may subsequently be easily sedimented and/or filtered (Vadasarukkai et al. 2011). Besides, the flocculator efficiency depends on hydraulic parameters, such as retention time and velocity gradient (G). The velocity gradient is fluid agitation representation. To avoid flocs disruption, the values of $\mathrm{G}$ must decrease as retention time increases, to avoid flocs disruption. Consequently, as the flow rate increases, it is expected higher velocity gradient in the structure and, thereby, impairs the equipment efficiency.
Perforated Tray-Type Flocculators (Fig. 1) were created in the 1970 s by Vianna (2019) as part of a prefabricated Water Treatment Plant (WTP). The equipment is composed of four vertical tanks with perforated trays, creating necessary agitation for flocculation. Each tank is designed to generate a different velocity gradient, decreasing from first to last, to minimize flocs rupture. Therefore, the diameters of the orifices increase from the first to the last tank. Thus, a larger cross-flow area in the last tank provides less turbulence, and consequently, preserving coagulated flocs. Furthermore, Brazilian Technical Regulation recommends that water velocity inside pipes for flocculated water is greater than $0.1 \mathrm{~m}$ $\mathrm{s}^{-1}$ and under $0.3 \mathrm{~m} \mathrm{~s}^{-1}$ (ABNT 1992).

The main advantage of the perforated tray-type vertical hydraulic flocculator is the possibility of installation in reduced areas. In addition, as it is a nationally used product, the equipment is in full operation in more than 200 Brazilian water treatment plants, mainly in the Minas Gerais state (Vianna 2019). Despite having been designed for the Brazilian 

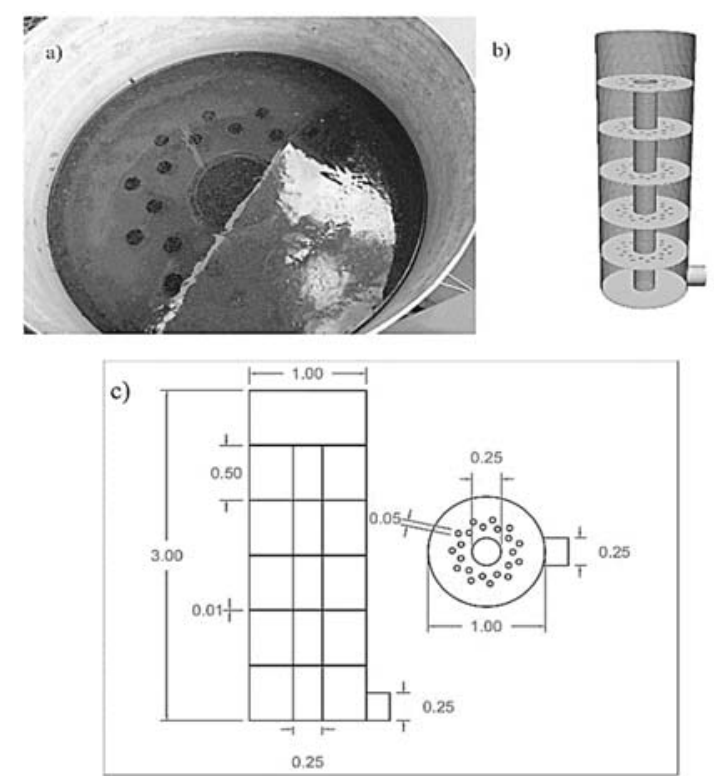

Fig. 1. Hydraulic perforated tray-type flocculator (a), computational domain (b), and geometry dimensions (in meters) (c).

demands, the possibility of increasing water treatment capacity in a reduced area of the WTS installation makes this type of flocculator highly adaptable to different locations, especially in small remote communities

There are not many experimental studies involving perforated tray-type flocculators in literature. Studies carried by experimental prototypes in a laboratory (Vianna et al. 2008) and real scale flocculators (Castro et al. 2018) estimated the mean $\mathrm{G}$ by head loss measurements and found much smaller agitation than projected. However, these results do not consider local $\mathrm{G}$, which can be highly elevated in orifices boundaries (Shah et al. 2012).

Experimental studies demand installation of prototypes, needs of specific materials, requiring time, and generating residuals. An interesting alternative to improve a project is the application of Computational Fluid Dynamics (CFD) tools to obtain a virtual response of the flow, before implementing experimental tests (Shah et al. 2012). The rise of computational tools has provided an advance in the Computer Assisted Engineering area. Thus, CFD tools have been increasingly utilized to analyze water and wastewater treatment plants (Alalm et al. 2016; Bridgeman et al. 2009; Samaras et al. 2010; Wang et al. 2014; Zhang et al. 2014).

Some studies in flocculators were performed in experimental mechanical prototypes, such as in jar test equipment (Bridgeman et al. 2008, 2010; Jarvis et al. 2004; Wang et al. 2014). Jarvis et al. (2004) have monitored particle size by analyzing the retention time and mixing velocities using different coagulants. When iron hydroxide was applied as a coagulant at a mixing rate of $75 \mathrm{rpm}$, the particle size tends to drop after 15 minutes of mixing.
Comparing these results to the computational simulations (Bridgeman et al. 2008), under the same conditions, it was observed that, in the mixing speed above $75 \mathrm{rpm}$, local velocity gradient values above $200 \mathrm{~s}^{-1}$ were observed. Thus, this may be considered a threshold value to avoid floc breakage.

Simulations of hydraulic flocculators using CFD (Bridgeman et al. 2010; Vadasarukkai et al. 2011) indicate the occurrence of flow recirculation and dead zones. Hence, it is possible to detect short circuits in these structures, and consequently, reducing the retention time. Vadasarukkai et al. (2011) have found values of $\mathrm{G}$ lower than $0.8 \mathrm{~s}^{-1}$ in $50 \%$ of the volume in the last tank structure.

In this way, the main objective of this study is to simulate the water flow in the last tank of a perforated tray-type flocculator via CFD (OpenFOAM, 2021). The last tank provides the formation of the final floc, and its proper functioning completes an appropriate flocs conglomeration. Ensuring ideal velocity gradients in this tank can provide flocs integrity.

A modification in the flocculator structure has been proposed and computationally simulated to increase the equipment's treatment capacity. The velocity gradient values were evaluated through comparison analysis when increasing flow rate and orifices number per tray. The effects of minimally invasive and low-cost modifications on velocity gradients and floc preservation were analyzed to improve solid removal efficiency in a drinking water treatment plant.

Despite being widely used in the Brazilian context, the perforated tray-type flocculator proposed by Vianna (2019) has never been studied using CFD resources. Thus, this study brings an essential contribution of innovation to the Brazilian context, with a strong possibility of expansion to other drinking water treatment contexts. 


\section{Material ANd Methods}

\subsection{Water Treatment Plant and Floccu- lator Description}

The water treatment plant simulated in this study was installed in 1991, and it is located on Federal University of Lavras (UFLA), in Minas Gerais Brazilian state, at coordinates $21^{\circ} 13^{\prime} 49.3$ " S and $44^{\circ} 58^{\prime} 39.8^{\prime \prime} \mathrm{W}$, whose altitude is in 893 meters above sea level. Currently, the average flow rate operation is $7.01 \mathrm{~s}^{-1}$. The maximum water treatment capacity of the station is approximately $91 \mathrm{~s}^{-1}$.

The water treatment brings autonomy to the university in terms of water supply, and the process follows seven steps: raw water catchment, preoxidation, coagulation, flocculation, decantation, filtration, and disinfection. Ferric chloride is used as a coagulant, followed by a rapid mixing generated by a Parshall flume. Next, the flocculator unit comprises four cylindrical tanks with five perforated trays each, where it is granted a decreasing velocity gradient. The orifices' diameter grows sequentially in each tank, reaching $0.05 \mathrm{~m}$ in the last flocculator, ensuring the velocity gradient reductions and preserving flocs' diameter for sedimentation in the next step.

The last tank was chosen to perform the CFD simulations in this study due to its importance in the flocculation process and the higher risk for floc rupture, guaranteeing the final preservation of the aggregated flocs before the decantation process.

The actual conditions in the current operation $(71 \mathrm{~s}$ $\left.{ }^{1}\right)$ were replicated and compared to their maximum capacity $\left(91 \mathrm{~s}^{-1}\right)$. As an alternative to increasing the flow rate, it was proposed to implement 11 extra orifices for each tray.

The fourth flocculation tank has a $1.0 \mathrm{~m}$ diameter cylindric and $3.3 \mathrm{~m}$ height. Inside the equipment, in its center, there is a $0.25 \mathrm{~m}$ diameter pipe. The trays are spaced every $0.5 \mathrm{~m}$. Each plate has 22 orifices, each of $0.05 \mathrm{~m}$ diameter (Fig. 1c). The tank was dimensioned and projected to guarantee an average velocity gradient of $20 \mathrm{~s}^{-1}$.

\subsection{Computational Fluid Dynamics}

\subsubsection{OpenFOAM}

OpenFOAM is a software created in 1993 by Henry Weller and Hrvoje Jasak at the Imperial College of London. The routines are a $\mathrm{C}++$ library composed of solvers with finite volume method to solve a continuous physical problem, as well as utilities to work in data manipulation (Ansoni and Seleghim 2016).

\subsubsection{Governing equations}

The general equation of continuity Eq. (1) is sufficient to describe a given flow of any specific quantity $(\boldsymbol{\phi})$ (Holzmann 2017):

$$
\frac{\partial}{\partial t} \rho \phi=-\nabla \cdot(\rho U \phi)+\nabla \cdot(D \nabla \phi)+S_{\phi}
$$

Where $\mathrm{D}$ is the diffusion coefficient (vector or scalar), $S_{\phi}$ is the source term, $\rho$ is the specific mass, and $U$ the velocity.

Equation (1) is the mathematical representation for fluid flow. It is described as the introductory physics for fluids laws, like mass conservation and Newton's second law, where the momentum is equal to the forces acting on a particle (Lira, 2014). By replacing the term $\phi$ into Eq. (1) by 1, the governing mass conservation Eq. (2) was obtained. The terms representing diffusion and source terms are annulled since the mass is not transferred through the diffusion process or into energy. For incompressible flow, the specific mass $(\rho)$ will be constant, which allows dividing both sides of the equation by $\rho$, obtaining Eq. (3) (Holzmann 2017).

$$
\begin{aligned}
& \frac{\partial}{\partial t} \rho=-\nabla \cdot(\rho U) \\
& \nabla \cdot U=0
\end{aligned}
$$

The forces acting on the fluid particles can be separated into two groups, i.e., surface forces or body forces. The first group is related to forces acting on the surface, such as pressure $(p)$ and shear stress $(\tau)$.

The second group covers the forces acting on the body, such as gravity force. It is common to use surface forces separately in the moment conservation equation and present the body forces as source terms. Thus, the moment conservation Eq. (4) is obtained by replacing the term $\phi$ for velocity $(U)$ in Eq. (3) (Holzmann, 2017).

$$
\frac{\partial}{\partial t} \rho U=-\nabla \cdot(\rho U \times U)+\nabla \cdot \tau-\nabla \cdot p+\rho g
$$

\subsubsection{Modeling turbulence by Standard k- $\varepsilon$ model}

In turbulent flows, the variables such as velocity, pressure, and temperature, oscillate in time and space. These fluctuations can be represented around an average value and in a defined interval time, called Reynolds-Average. Among those, there are turbulence models able to measure these oscillations, whose characteristics must include precision, full application, and reduced computational cost. Thus, the k- $\varepsilon$ model, besides having such attributes, is the most used and validated in the literature. Two equations are solved, one for $\mathrm{k}$ and another for $\varepsilon$, where $k$ is the turbulent kinetic energy, in $\mathrm{m}^{2} \mathrm{~s}^{-2}$, and $\varepsilon$ the rate of kinetic energy dissipation, in $\mathrm{m}^{2} \mathrm{~s}^{-3}$. These can be used to find the turbulent viscosity $\left(\mu_{t}\right)$, as shown in Eq. (5), where $C_{\mu}$ is a dimensionless constant (Versteeg and Malalasekera 2007).

$$
\mu_{t}=C \rho \vartheta l=\rho C_{\mu} \frac{K^{2}}{\varepsilon}
$$

Thus, the equations that will then define the values of $k$ and $\varepsilon$ will be: 


$$
\begin{aligned}
\frac{\partial(\rho k)}{\partial t}+\nabla \cdot(\rho k U)= & \nabla \cdot\left[\frac{\mu_{t}}{\sigma_{k}} \nabla k\right]+ \\
2 \mu_{t} S_{i j}^{2}-\rho \varepsilon & \\
\frac{\partial(\rho \varepsilon)}{\partial t}+\nabla \cdot(\rho \varepsilon U)= & \nabla \cdot\left[\frac{\mu_{t}}{\sigma_{\varepsilon}} \nabla \varepsilon\right]+C_{1 \varepsilon} \frac{\epsilon}{k} 2 \mu_{t} S_{i j}^{2} \\
& -C_{2 \varepsilon} \rho \frac{\varepsilon^{2}}{k}
\end{aligned}
$$

The term $S_{i j}$ is represented in Eq. (8), given by:

$$
S_{i j}=S_{x y}=\frac{1}{2}\left[\frac{\partial U x}{\partial y}+\frac{\partial U_{y}}{\partial x}\right]
$$

The five constants $C_{\mu}, \sigma_{k}, \sigma_{\varepsilon}, C_{1 \varepsilon}$, and $C_{2 \varepsilon}$ have standardized values by default. The standard constants are defined by Eq. (9). These were validated for an orifice simulation experiment (Shah et al. 2012).

$$
\begin{aligned}
& C_{\mu}=0,09 ; \sigma_{k}=1,00 ; \sigma_{\varepsilon}=1,30 ; \\
& C_{1 \varepsilon}=1,44 ; C_{2 \varepsilon}=1,92
\end{aligned}
$$

As discussed by Bridgerman et al. (2009) and Vadasarukkai et al. (2011), the k- $\varepsilon$ turbulence model is simple, robust, and well-established. This turbulence model can be applied for flocculation tank computational simulations to evaluate the gradient analysis, which is more meaningful to guarantee the flocs integrity.

Moreover, under steady-state conditions, the distribution of $\mathrm{k}$ and $\varepsilon$ at various locations in the tank can be predicted by solving the corresponding transport Eqs. (6) and (7) of the standard k- $\varepsilon$ model. Then, the local velocity gradient can be evaluated for each of the control volumes.

\subsubsection{Mesh generation and conditions}

In this study, the problem geometry domain was created on a scale of $1: 1$, with a total volume of 2.24 $\mathrm{m}^{3}$. Free Gmsh software was applied to generate an unstructured, three-dimensional, and tetrahedronshaped mesh for the geometry with 22 orifices. The number of elements of the generated meshes can be seen in Table 1.

Table 1. Descriptions of meshes used for convergence tests.

\begin{tabular}{|c|c|}
\hline \multirow{2}{*}{$\begin{array}{c}\text { Mesh } \\
\text { identification }\end{array}$} & Flocculator with 22 orifices \\
\cline { 2 - 2 } & Number of elements \\
\hline Mesh 1 & 215228 \\
\hline Mesh 2 & 373313 \\
\hline Mesh 3 & 602963 \\
\hline Mesh 4 & 1029250 \\
\hline
\end{tabular}

\subsubsection{Boundary conditions}

An initial approximation for velocity was assigned to the entire internal structure (internalField) for a value of $0 \mathrm{~m} \mathrm{~s}^{-1}$. For the boundary conditions (boundaryField), the velocity structure entrance was calculated through the flow rate and crosssectional area. Since the pipe is $0.25 \mathrm{~m}$ in diameter, the velocity at the inlet pipe for a flow rate of $7.01 \mathrm{~s}^{-1}$ is approximately $0.14 \mathrm{~m} \mathrm{~s}^{-1}$.

By increasing the flow rate to $9.01 \mathrm{~s}^{-1}$, the velocity changes to $0.18 \mathrm{~m} \mathrm{~s}^{-1}$. These values were assigned using the fixedValue condition of OpenFOAM, which assures a constant value on that surface. The geometry walls have noSlip condition, which assigns the velocity equal to $0 \mathrm{~m} \mathrm{~s}^{-1}$. The output is zeroGradient, which indicates that the gradient is null, i.e. the direction will remain perpendicular to the surface. For the upper surface of the flocculator, the slip condition was adopted, allowing to perform the behavior of the water surface. This setting assigns a null velocity value to the surface and uses zeroGradient in the tangential components. This configuration is commonly applied in other studies (Bridgeman et al. 2008, 2010; Vadasarukkai et al. 2011).

The structure outlet pressure was assigned according to the value of $29.4 \mathrm{kPa}$, and the specific mass of the water at $20^{\circ} \mathrm{C}, 1.0 \mathrm{~g} \mathrm{~cm}^{-3}$. The other surfaces of the geometry were given the zeroGradient condition.

The initial approximations for $\varepsilon$ and $k$ values were calculated using Eqs. (10) and (11).

$$
\begin{aligned}
& k=\frac{3}{2}\left(I\left|U_{\text {ref }}\right|\right)^{2} \\
& \varepsilon=\frac{C_{\mu}{ }^{0,75} k^{1,5}}{L}
\end{aligned}
$$

where $I$ is the turbulence intensity $(10 \%), C_{\mu}$ is a constant equal to 0.09 , and $L$ is the reference length (Salgado 2006)

During the flocculation process, the actual flow in the tank is a multiphase flow, composed of water and flocs formed by dust and several kinds of organic residues.

According to Richter (2009), as the flake grows, its density decreases. Thus, the flocs become more subject to the hydrodynamic shear stress generated by velocity gradients. Considering the process simulated in the last flocculation tank, maintaining the stability of the floc formed becomes more relevant for the efficiency of the processes than simulating the interaction between the flocs that are already fully formed. The flocs do not exceed the average size of $0.44 \mathrm{~mm}$ (Tambo and Hozumi, 1979), with passive behavior. Thus, the computational modelling can be simplified as a single-phase model. The velocity gradient analysis is enough to predict the flocs integrity.

Thus, the flow inside the flocculation equipment can be considered stationary, incompressible, and singlephase. Therefore, the simpleFoam routine implemented in OpenFOAM was applied as the problem solver. The simpleFoam routine applied the SIMPLE (Semi-Implicit Method for Pressure Linked Equations) algorithm. It was necessary to apply a low relaxation factor to avoid floating point exception, run the simulation more stable, and allow the convergence of the problem (Holzmann 2017). In this study, a relaxation factor of 0.1 was used, which ensured the convergence of the simulations. 
The simulations start with all velocities set zero, whilst $\mathrm{k}$ and $\varepsilon$ were set to $1.44 \mathrm{e}^{-4} \mathrm{~m}^{2} \mathrm{~s}^{-2}$ and $3 \mathrm{e}^{-5}$ $\mathrm{m}^{2} \mathrm{~s}^{-2}$, respectively. For all simulations, the convergence criteria were set $1 \mathrm{e}^{-4}$ for pressure and set $1 \mathrm{e}^{-5}$ for velocity and turbulent components. The percentage of relative error $(\% \mathrm{ER})$ was evaluated by:

$$
\begin{aligned}
\% \mathrm{ER}_{\mathrm{j}} & =\frac{\left|\mathrm{X}_{\mathrm{j}}^{\mathrm{i}}-\mathrm{X}_{\mathrm{j}}^{\mathrm{ref}}\right|}{\left|\mathrm{X}_{\mathrm{j}}^{\mathrm{ref}}\right|} \times 100 \\
M E R_{j} & =\Sigma_{j=1}^{N} \frac{E R_{j}}{N}
\end{aligned}
$$

where $X$ is the velocity magnitude at point $j, N$ is the number of cells in the mesh, and the indexes $i$ and ref refer to coarse and fine mesh, respectively. $M E R i$ is the factor of safety. As indicated in Roche and Patrick (1998), the MERi was set equal to 3.

The simulations were carried out in parallel (24 threads) on a cluster running under CentOS Linux release 7.4.1708 and OpenFoam v7 [OpenFOAM, 2021]. Table 2 shows the cluster specifications applied to perform the simulations.

Table 2. Workstation specifications.

\begin{tabular}{|c|c|}
\hline Cluster & Workstation specifications \\
\hline Head Node & $\begin{array}{c}\text { Intel(R) Xeon(R) CPU X5670 } \\
\text { 2.93GHz; RAM: 16GB; HD 1TB }\end{array}$ \\
\hline $\begin{array}{c}\text { 9 compute } \\
\text { nodes }\end{array}$ & $\begin{array}{c}\text { Intel(R) Xeon(R) CPU X5660 } \\
2.80 \mathrm{GHz} ; \text { RAM: 12 GB; HD } \\
500 \mathrm{~GB}\end{array}$ \\
\hline
\end{tabular}

The results for the performance of the four meshes tested are shown in Table 3.

Table 3. Convergence tests results

\begin{tabular}{|c|c|c|c|}
\hline Mesh & $\begin{array}{c}\text { Average } \\
\text { relative } \\
\text { error }\end{array}$ & $\begin{array}{c}\text { Processing } \\
\text { time[s] }\end{array}$ & $\begin{array}{c}\text { Number } \\
\text { of } \\
\text { iterations }\end{array}$ \\
\hline Mesh 1 & $23.78 \%$ & 571.00 & 2795 \\
\hline Mesh 2 & $17.55 \%$ & 2974.72 & 2821 \\
\hline Mesh 3 & $14.12 \%$ & 33626.00 & 62812 \\
\hline Mesh 4 & $\begin{array}{c}\text { Reference } \\
\text { mesh }\end{array}$ & 50216.00 & 52542 \\
\hline
\end{tabular}

The increase in grid density between the Mesh 3 and Mesh 4 led to an increase in computation time of about 5 hours to achieve a converged solution. It was concluded that the increased computational effort was not reflected in a corresponding increase in the accuracy of results. This can be seen in Fig. 3 , where the velocity magnitude was plotted over a line inside the flocculator, showed in Fig. 2.

In this way, the best cost-benefit in the analyzed meshes was obtained by mesh 3 . This mesh was applied in the subsequent simulations.

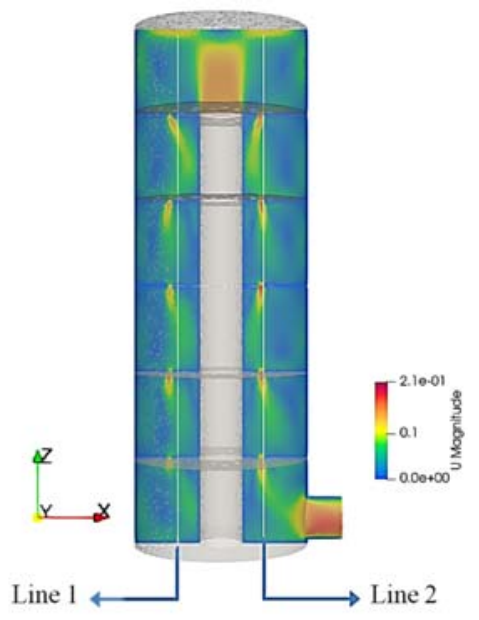

Fig. 2. Lines defined in the flocculator geometry for analysis of mesh convergence.

\subsection{Velocity Gradient}

The velocity gradient $(\mathrm{G})$ is an essential factor for the formation of flocs because it defines the intensity of the mixture in a flow. To calculate it locally $\left(G_{l}\right)$, the energy dissipation rate (Camp and Stein 1943) should be included and calculated by Eq. (14).

$$
G_{l}=\sqrt{\frac{\varepsilon}{\vartheta}}
$$

Since dissipation energy $(\varepsilon)$ is in $\mathrm{m}^{2} \mathrm{~s}^{-3}$ and the kinematic viscosity $(\vartheta)$ in $\mathrm{m}^{2} \mathrm{~s}^{-1}$, the local velocity gradient $\left(G_{l}\right)$ will be given in $\mathrm{s}^{-1}$.

For a hydraulic flocculator, the velocity gradient value is calculated as a function of the head loss $\left(h_{f}\right)$ in meters, the retention time $(T)$ in seconds, kinematic viscosity $(\vartheta)$ in $\mathrm{m}^{2} \mathrm{~s}^{-1}$ and the gravity acceleration $(g)$ in $\mathrm{m} \mathrm{s}^{-2}$ (Bridgeman et al. 2010) by Eq. (15).

$$
\mathrm{G}=\sqrt{\frac{g h_{f}}{\vartheta T}}
$$

The velocity gradient inside a flocculator is not uniform, so an average or overall value for $\mathrm{G}$ may not accurately represent the flow conditions. However, it is possible to use numerical methods to calculate the gradient at each point of the control volume (Bridgeman et al. 2010).

\section{RESULTS}

To analyze the velocity gradients inside the flocculator, two initial simulations were executed. In the first simulation, the tank has an operating $71 \mathrm{~s}^{-1}$ flow and four plates with 22 orifices each. With the increased demand for treated water, the second simulates the flocculator while operating at the maximum capacity $\left(91 \mathrm{~s}^{-1}\right)$. Thus, the third and final simulation was to evaluate how a low-cost, noninvasive change could improve the flocculator performance in terms of hydraulic efficiency. As a 


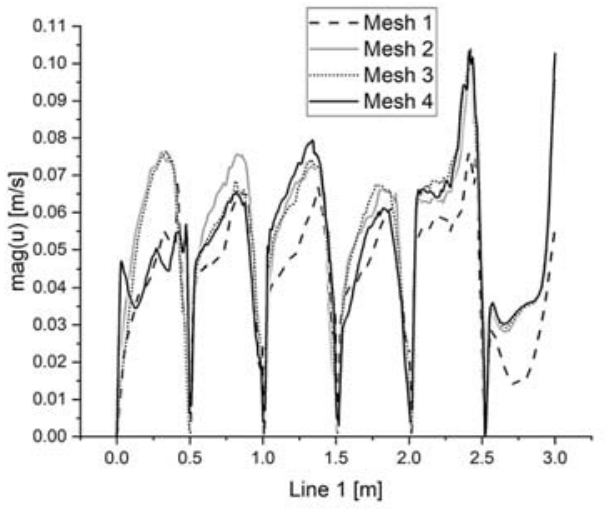

(a)

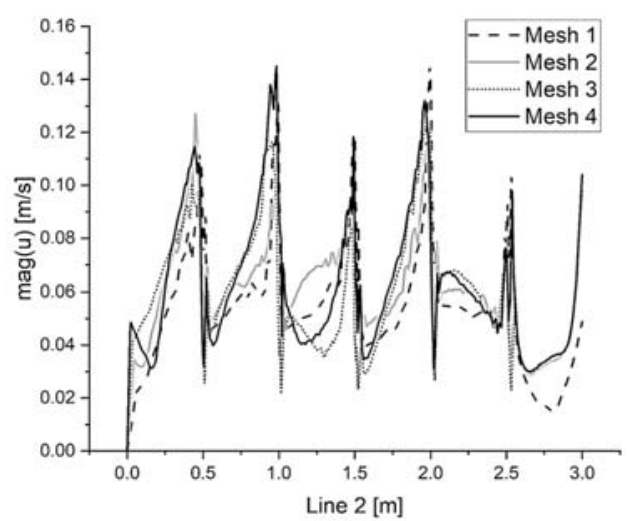

(b)

Fig. 3. Influence of mesh quality over the magnitude of velocities over line 1 (a) and line 2 (b).

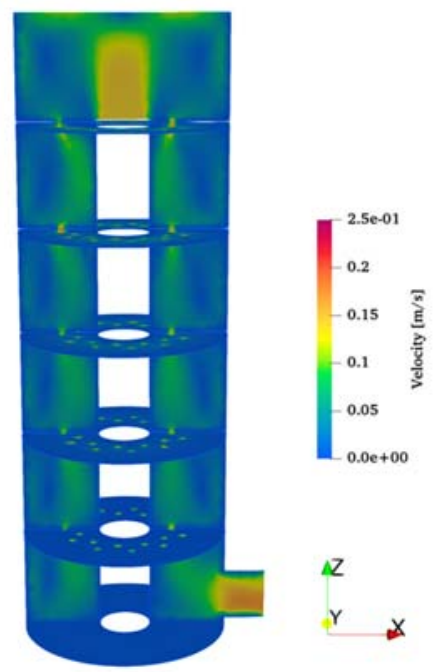

Fig. 4. Velocity in the flocculator for $7.0 \mathrm{I}$ $\mathrm{S}^{-1}$ flow rate in 22 orifices per plate.

result, the number of orifices in each plate was changed from 22 to 33 . Detailed results are presented in this section.

\subsection{Velocity field analysis}

The first simulation was performed for the flocculator with 22 orifices and flow $71 \mathrm{~s}^{-1}$ flow rate. The velocity in the last flocculation tank in the longitudinal section is shown in Fig. 4. It was observed that the velocity in the structure does not exceed values of $0.3 \mathrm{~m} \mathrm{~s}^{-1}$, as required by the Brazilian standard (ABNT 1992). However, most of the domain has velocity values lower than $0.05 \mathrm{~m} \mathrm{~s}^{-1}$, which is favorable for the sedimentation of flocs, not intended at this stage of treatment.

In Fig. 5, the velocity vectors generated by the flow inside the structure can be observed. Notice that the flow exhibits recirculation behavior, where the fluid near the bottom plate tends to return to the top plate at the edges of the tank in the 22 orifices simulations
(Fig. 5a and 5b). Then, it is possible to identify the dead zones formation qualitatively. Such conditions are consistent with other studies about hydraulic flocculators (Bridgeman et al. 2010; Vadasarukkai et al. 2011), where standard dead zones and short circuits were identified. This type of recirculation is an unwanted process in flocculators, causing retention time reduction.

When this water treatment plant operates at lower flow rates, unwanted sedimentation can be observed in the flocculator trays, requiring constant equipment cleaning. The sedimentation is a consequence of dead zones occurrence in this unit.

Figure $5 \mathrm{~b}$ shows the simulation results while increasing the flow rate from $71 \mathrm{~s}^{-1}$ to $91 \mathrm{~s}^{-1}$ in a flocculator with 22 orifices in each tray. Observing the velocity vectors for this flow rate simulation makes it possible to identify a few differences in flow behavior. However, dead zones regions continue to occur in some parts of the volume. The most relevant difference between the two flow conditions is the increase in the magnitude of velocity. As the fluid passes throughout the orifices and conduction tubes to the following structure, these values reached $0.2 \mathrm{~m} \mathrm{~s}^{-1}$. The water velocity in the flocculator did not exceed $0.3 \mathrm{~m} \mathrm{~s}^{-1}$ in this case.

As the flow increases, the theoretical retention time of the structure decreases due to the volume to flow ratio, which is now 17 minutes counting the four tanks. Once there is no observed improvement in the short-circuits formed, this condition can interfere in the flocculation efficiency. The flocs may not reach desirable diameters to guarantee the following decantation process, thus overloading the filters.

To propose improvements in the flocculation conditions at a flow rate of $9.01 \mathrm{~s}^{-1}$, this study proposes solutions that would be easy to implement, avoiding the complete tank replacement. The proposal is to make the most of the old configuration, reducing environmental impacts and damage to the project. In this way, the flocculator trays were redesigned, increasing the number of orifices in each tray. It was suggested to add another 11 orifices to the existing 
a)

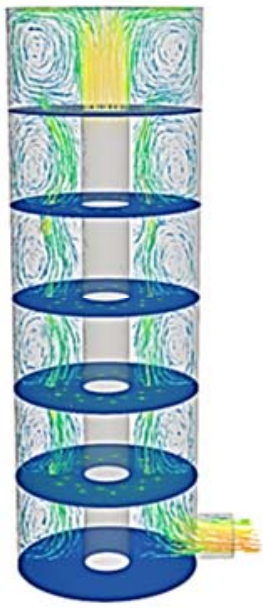

b)

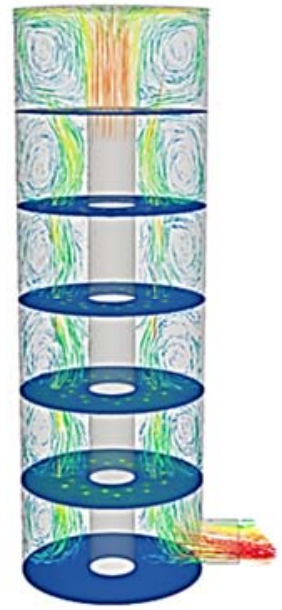

c)

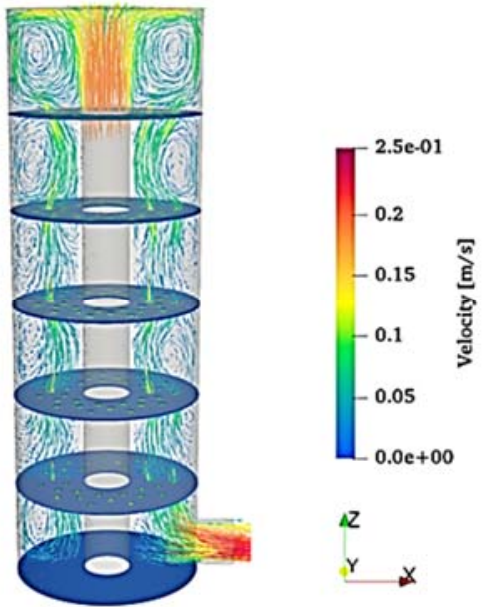

Fig. 5. Velocity vectors with $7.0 \mathrm{~L} \mathrm{~s}^{-1}\left(\right.$ a), $9,0 \mathrm{~L} \mathrm{~s}^{-1}$ (b) in 22 orifices, and $9,0 \mathrm{~L} \mathrm{~s}^{-1}$ with 33 orifices per plate (c).

tray, totalizing 33 orifices. The goal is to increase the flow cross-section area, reducing the Reynolds number and velocity gradient in the orifice.

Figure $5 \mathrm{c}$ shows a moderated improvement in the short circuits between trays due to the newly added orifices, which allows a better distribution between one compartment and another. However, the dead zones appear to be occupying a smaller space in the structure than observed in the previous simulations. This condition can improve the distribution of the particles, increase the real retention time, and reduce flocs sedimentation inside these structures.

\subsection{Velocity Gradient}

Values of $\varepsilon$ from turbulence model k- $\varepsilon$ were applied in the equation of the local velocity gradient (Eq. (14)). When calculated in the whole mesh, it was found values of $G_{l}$ at each point of the control volume. Thus, it was possible to provide a quantitative analysis of the turbulence behaviors in the structure.

The spatial projection of $G_{l}$ can be seen in Fig. 6 a. It is possible to notice that closer to the orifices the values are greater than $30 \mathrm{~s}^{-1}$, reaching $150 \mathrm{~s}^{-1}$. These values are higher than the expected by which the structure was designed $\left(20 \mathrm{~s}^{-1}\right)$. Also, smaller $G_{l}$ values in a larger portion of the tank were observed in another study performed for hydraulic flocculators (Vadasarukkai et al. 2011). This condition causes the average velocity gradient of the structure to be $8.55 \mathrm{~s}^{-1}$, which would explain the results found by Castro et al. (2018), where experimental analysis of $\mathrm{G}$ indicated smaller agitation than projected.

Considering the importance of the $G_{l}$ analysis for the flocculation process, it is essential to analyze the gradient values behavior when fluid passes through the tray orifices. Thus, in Fig. $6 \mathrm{~b}$ and $6 \mathrm{c}$, the orifices of a single tray can be observed by a cross-section cut and its respective histogram of the $G_{l}$ distribution.

Figure $6 \mathrm{~b}$ shows most occurrences of local velocity gradient between 50 and $70 \mathrm{~s}^{-1}$ when passing through the orifice, with the maximum value close to $145 \mathrm{~s}^{-1}$. These values are not adequate for the last flocculation tank. Higher intensity of $G_{l}$ in this water treatment step can promote flocs breakage and prejudice the following decantation step in water treatment.

As the flow rate increases, the turbulence in the structure is also intensified. Therefore, it was necessary to understand the effects of a flow rate increase in the values of $G_{l}$, considering the treatment system at a higher capacity. By simulating a flow rate of $9.01 \mathrm{~s}^{-1}$ (Fig. 7a), an increase in velocity gradient was observed as the fluid passed through the orifices.

Observing the histogram (Fig. 7b), it is noticeable that there was a significant increase in the velocity gradient there. For a higher flow rate $\left(9.01 \mathrm{~s}^{-1}\right)$, local velocity gradients occur mostly between 80 and $100 \mathrm{~s}^{-1}$, reaching a maximum value close to $200 \mathrm{~s}^{-1}$. Thus, if the increased demand for treated water is met through changes in flow rates, the efficiency of the flocculation process will be strongly jeopardized. Bridgeman et al. (2008) found a threshold for flocs breakage of $200 \mathrm{~s}^{-1}$ for local velocity gradient after 15 minutes in a jar test CFD experiment for iron hydroxide precipitate flocs. Hence, at full treatment capacity, it will not be possible to guarantee the flocs preservation.

The solution proposed for the higher velocity gradient problem was to increase each flocculation tray's orifices number. The modification is minimally invasive, cost-effective, and easy to implement in the flocculation tank configuration with removable trays. Figure 8a presents the local velocity gradient for the modified geometry, where the number of perforated tray orifices was increased from 22 to 33 . It is observed that the value of $G_{l}$ reduced considerably compared to Fig. 7. It is possible to notice that there is a turbulence reduction due to the increase in crossflow area. As a result, the external orifices have lower 


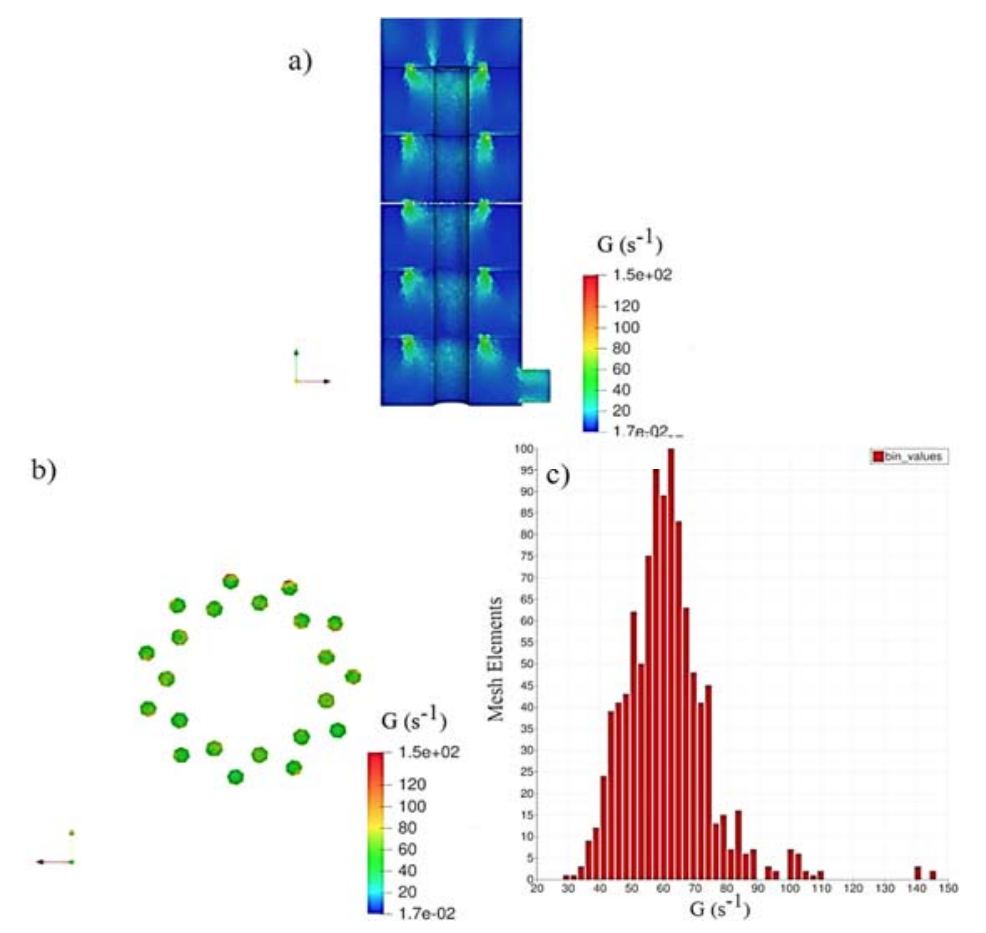

Fig. 6. $G_{l}$ for $7.0 \mathrm{~L} \mathrm{~s}^{-1}$ flow rate in 22 orifices per plate (a), cross-section $2 \mathrm{D}$ cut of the perforated tray (b) and its respective $G_{l}$ histogram distribution (c).

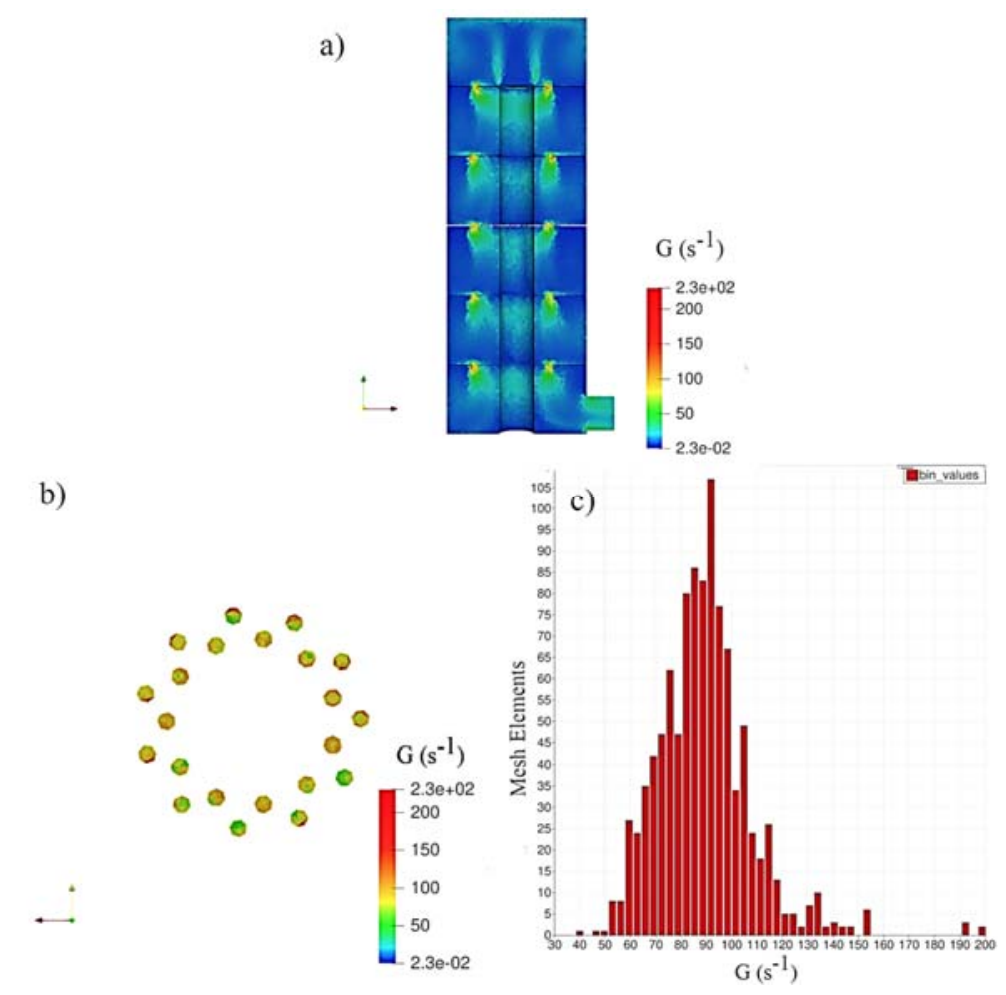

Fig. 7. $G_{l}$ for $9.0 \mathrm{~L} \mathrm{~s}^{-1}$ in 22 orifices per plate (a), cross-section 2D cut of the perforated tray (b) and its respective $G_{l}$ histogram distribution (c). 


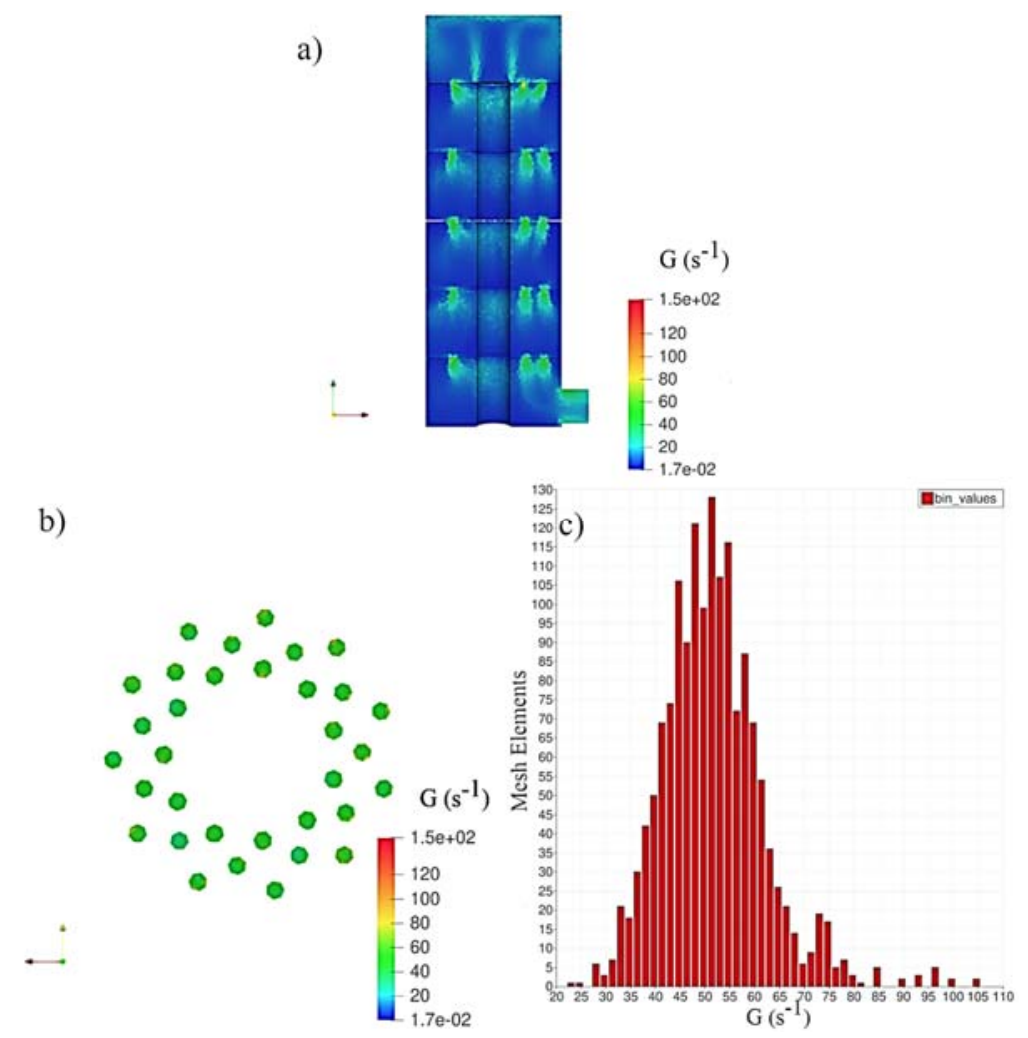

Fig. 8. $G_{l}$ for $9.0 \mathrm{~L} \mathrm{~s}^{-1}$ in 33 orifices per plate (a) cross-section $2 \mathrm{D}$ cut of the perforated tray (b) and its respective $G_{l}$ histogram distribution (c).

$\mathrm{G}_{1}$ values. The low fluid velocity may justify this fact in this section of the flow, as the turbulence depends on the interaction of the fluid velocity with the wall. This condition explains the occurrence of higher $G_{l}$ values at the edges of each orifice, where the fluid is affected by the wall contact.

Plotting a 2-dimensional plane on the trays (Fig. $8 \mathrm{~b}$ ), it is possible to evaluate the distribution of $G_{l}$ as it passes through the orifices when the flocculator geometry is modified. Here, the values of $G_{l}$ occurred mostly between approximately 40 and 60 $\mathrm{s}^{-1}$, with a maximum value of $105 \mathrm{~s}^{-1}$. Therefore, there was an improvement in the turbulence conditions since the configuration of the perforated tray does not generate high values for $G_{l}$. Furthermore, the flow simulation for this geometry configuration provided the most favorable situation for the flocculation process in the last tank. Thus, the new geometry configuration ensures smoother mixing rates and the formed flocs preservation.

To complete the quantitative velocity gradient parsing, Table 4 presents a statistical analysis. The simulations performed at current conditions $\left(7 \mathrm{~s} \mathrm{~s}^{-1}\right)$ were compared for the flows of $91 \mathrm{~s} \mathrm{~s}^{-1}$ with 22 orifices and 33 orifices. Measuring the position and dispersion of the values of $G_{l}$, it was possible to notice that there is a considerable reduction in all variables when structural modifications are made. The mean $G_{l}$ decreased from 12.48 to $9.51 \mathrm{~s} \mathrm{~s}^{-1}$, and the standard deviation was reduced from 12.37 to
$8.54 \mathrm{~s}^{-1}$. This reduction shows that the $G_{l}$ values are close to average in the perforated tray with 33 orifices. The occurrence of $95 \%$ percentile and $99 \%$ percentile of $G_{l}$ in the mesh also reduced considerably. The values were from 33.14 to $25.24 \mathrm{~s}^{-1}$ and from 73.46 to $46.76 \mathrm{~s}^{-1}$, respectively.

Table 4. Velocity gradient statistical analysis using position and dispersion measurements

\begin{tabular}{|c|c|c|c|}
\hline \multirow{2}{*}{ Statistics } & \multicolumn{3}{|c|}{$G\left(s^{-1}\right)$} \\
\hline & $\begin{array}{c}7.0 \mathrm{~L} \mathrm{~s}^{-1} \\
\text { (22 orifices) }\end{array}$ & $\begin{array}{c}9.0 \mathrm{~L} \mathrm{~s}^{-1} \\
(22 \text { orifices) }\end{array}$ & $\begin{array}{c}9.0 \mathrm{~L} \mathrm{~s}^{-1} \\
\text { (33 orifices) }\end{array}$ \\
\hline Average & 8.55 & 12.48 & 9.51 \\
\hline $\begin{array}{l}\text { Standard } \\
\text { deviation }\end{array}$ & 8.50 & 12.37 & 8.54 \\
\hline $\begin{array}{c}5 \% \\
\text { percentile }\end{array}$ & 2.48 & 3.66 & 2.87 \\
\hline $\begin{array}{c}50 \% \\
\text { percentile }\end{array}$ & 6.08 & 8.93 & 6.85 \\
\hline $\begin{array}{c}95 \% \\
\text { percentile }\end{array}$ & 22.77 & 33.14 & 25.24 \\
\hline $\begin{array}{c}99 \% \\
\text { percentile }\end{array}$ & 50.34 & 73.46 & 46.76 \\
\hline Maximum & 183.83 & 265.68 & 157.49 \\
\hline
\end{tabular}

Thus, a similar condition observed when isolating only the orifices tray is also observed when the entire flocculator structure is analyzed. Comparing simulations results between the flocculators with 
increased flow rate $\left(91 \mathrm{~s}^{-1}\right)$ and 33 orifices (Fig. 8a) and reduced flow rate $\left(7 \mathrm{~s} \mathrm{~s}^{-1}\right)$ with 22 orifices (Fig. $6 \mathrm{a})$, it is possible to observe that the dispersion and position measurements reach very close values. Thus, it is possible to ensure the flocs preservation despite the necessary increase in flow rate.

The cumulative frequency distribution analysis (Fig. 9), as discussed in other studies (Bridgeman et al. 2010; Vadasarukkai et al. 2011), allows comparing the changing of $\mathrm{G}_{1}$ values for the performed cases in this study. As the flow rate increases, it is possible to notice a curve shift to the right, showing an increase in the structure's velocity gradient. However, by increasing the number of orifices, it is possible to observe that the curve shifts back to the left. Therefore, it is possible to conclude that when increasing the flow rate and the number of orifices from 22 to 33 , the turbulence in the structure will be similar to those at a lower flow rate (7 $1 \mathrm{~s} \mathrm{~s}^{-1}$ and 22 orifices). This flow behavior contributes to the formation of the floc in the transition between the flocculators compartments. The cumulative frequency distribution results shown in Fig. 9 show the same behavior as those found by Vadasarukkai et al. (2011).

The shear stress analysis can also provide parameters for flocs preservation. Table 5 shows the shear stress values for the two operating flow rates of the flocculator and the two simulated geometric conditions. It is possible to observe that the shear stress values increase with increasing flow rate from $7 \mathrm{~L} \mathrm{~s}^{-1}$ to $9 \mathrm{~L} \mathrm{~s}^{-1}$ in the flocculator. This increase can cause flocs disruption, impairing the decanting process. With the proposed geometric modification from 22 to 33 orifices, it is possible to notice the drop in the shear stress values, ensuring the integrity of the flocs already fully formed in the last flocculation tank.

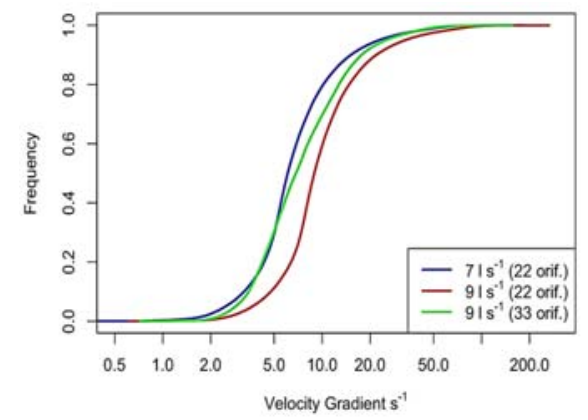

Fig. 9. Cumulative frequency distribution as a function of $\mathbf{G}_{\mathbf{l}}$.

Table 5. Shear Stress analysis $\left(\mathrm{m}^{2} \mathrm{~s}^{-2}\right)$ for flocculator tank with 22 and 33 orifices per plate

\begin{tabular}{|c|c|c|}
\hline Flow rate & 22 orifices & 33 orifices \\
\hline $7 \mathrm{~m} \mathrm{~s}^{-1}$ & $9.70 \mathrm{e}^{-4}$ & $6.70 \mathrm{e}^{-4}$ \\
\hline $9 \mathrm{~m} \mathrm{~s}^{-1}$ & $1.60 \mathrm{e}^{-3}$ & $1.20 \mathrm{e}^{-3}$ \\
\hline
\end{tabular}

\section{Conclusions}

The process of flocculation by controlled agitation and coagulation is crucial for the proper functioning of a drinking water treatment plant. Improving this process ensures better agglomeration of the colloid found naturally in raw water collected from nature. Furthermore, it also contributes to improving the performance of decantation, the following stage of treatment, and, consequently increases solid removal efficiency

In this study, Computational Fluid Dynamics was applied to simulate the flow of water in the last tank of a perforated tray-type flocculator using a CFD opensource software: OpenFOAM. A modification in the flocculator structure has been proposed and computationally performed to increase the equipment's treatment capacity. As a low-cost and minimally invasive intervention, the number of orifices was increased from 22 to 33 . The velocity gradient values were evaluated through comparison analysis when increasing flow rate and orifices number per tray.

Before the geometry modification, the numerical results indicated velocity gradient values above the projected, due to the occurrence of areas with greater turbulence within the wall of the orifice. Dead zones were found at the perforated tray extremities, which resulted in unnecessary decantation of flocs. Thus, there is a formation of short circuits. The short circuit occurrence reduces the retention time of the flocs in the flocculation process, impairing the efficiency of the equipment.

To increase flocculator processing capacity, by increasing the flow rate from $71 \mathrm{~s}^{-1}$ to $91 \mathrm{~s}^{-1}$, by the simulation for the geometry with 22 orifices configuration, it was possible to identify a significant increase of $\mathrm{G}_{1}$ in the orifices, causing flocs breakage. Therefore, the proposed solution was to increase the number of orifices from 22 to 33. Hence, obtained values for $\mathrm{G}_{1}$ were similar to the flocculator with a flow rate of $71 \mathrm{~s}^{-1}$. Consequently, this geometry improvement upgraded the equipment's efficiency by a simple change in geometry despite the increased flow rate.

The results obtained for the analysis of velocity gradients agree with the results given by Bridgeman et al. (2010) and Vadasarukkai et al. (2011).

In conclusion, analyzing the efficiency of flocculators through Computational Fluid Dynamics can be an important way to optimize this type of equipment and the Water Treatment Plant as a whole. Flocculators working at a suitable velocity gradient provide better flocs aggregation. Consequently, they also improve following processes, such as decantation and filtration, for example, removing larger amounts of solids and producing better quality drinking water. Thus, the CFD simulations performed in this study meet increased demand for treated water for the increasing population through an affordable and non-invasive optimization process. 
P. A. Melo et al. / JAFM, Vol. 15, No. 2, pp. 387-397, 2022.

\section{ACKNOWLEDGEMENTS}

The authors would like to thank the $\mathrm{CNPq}$ (Conselho Nacional de Desenvolvimento Científico e Tecnológico), grant 428792/2018-9, for the financial support. This research was carried out using the computational resources of the Center for Mathematical Sciences Applied to Industry (CeMEAI) funded by FAPESP (grant 2013/07375$0)$.

\section{REFERENCES}

ABNT. (1992). NBR 12216: Project of water treatment plant for public supply, Brazilian Association of Technical Norms, Brasilia, Brazil.

Alalm, M. G., M. Nasr and S. Ookawara (2016). Assessment of a novel spiral hydraulic flocculation/sedimentation system by CFD simulation, fuzzy inference system, and response surface methodology. Separation and Purification Technology 169, 137-150.

Ansoni, J. L. and P. Seleghim (2016). Optimal industrial reactor design: Development of a multiobjective optimization method based on a posteriori performance parameters calculated from CFD flow solutions. Advances in Engineering Software, available.

Bridgeman, J., B. Jefferson and S. A. Parsons (2009). Computational Fluid Dynamics Modelling of Flocculation in Water Treatment: A Review. Engineering Applications of Computational Fluid Mechanics.

Bridgeman, J., B. Jefferson and S. A. Parsons (2010), The development and application of CFD models for water treatment flocculators. Advances in Engineering Software.

Bridgeman, J., B. Jefferson and S. Parsons (2008). Assessing floc strength using CFD to improve organics removal. Chemical Engineering Research and Design.

Camp, T. and P. Stein (1943). Velocity gradients and internal work in fluid motion. Boston Society of Civil Engineers.

Castro, L. V., M. Rocha Vianna, C. de O. Ribeiro and L. S. Altair (2018). The influence of submerged orifice discharge coefficients in project of perforated tray-type hydraulic flocculator in water treatment plants. AIDIS Journal of Engineering and Environmental Sciences: Research, Development and Practice 11(1), 36-48.

Holzmann, T. (2017). Mathematics, Numerics, Derivations and OpenFOAM. Configurable Distributed Systems, 1992. International Workshop On.

Jarvis, P., B. Jefferson and S. Parsons (2004).The duplicity of floc strength. Water Science and Technology.

Lira V. M. P. (2014) Numerical Modeling of a $90^{\circ}$
Open channel Confluence Flow Using Openfoam CFD. M.Sc. dissertation, Universidade Federal de Minas Gerais, Belo Horizonte, Brazil.

OpenFOAM (2021), The OpenFOAM Foundation Ltd, available at: https://openfoam.org/version/7, Accessed 16.06.21.

Richter, C. A. (2009). Água: Método e Tecnologia de Tratamento. São Paulo. Blucher.

Salgado, A. L. (2006), Hydrodynamic Characterization of Chicaned Hydraulic Flocculators Through Three-Dimensional Numerical Simulation, M.Sc. Dissertation, Universidade Federal do Espírito Santo, Vitória, Brazil

Samaras, K., A. Zouboulis, T. Karapantsios and M. Kostoglou (2010). A CFD-based simulation study of a large scale flocculation tank for potable water treatment. Chemical Engineering Journal 162, 208-216.

Shah, M. S., J. B. Joshi, A. S. Kalsi, C. S. R. Prasad and D. S. Shukla (2012). Analysis of flow through an orifice meter: CFD simulation. Chemical Engineering Science 71, 400-309.

Tambo, N. and H. Hozumi (1979). Physical aspect of flocculation process-II. Contact flocculation. Water Research 13(5), 441-448.

Tambo, N. and H. Hozumi (1979). Physical characteristics of flocs-II. Strength of floc. Water Research.

Vadasarukkai, Y. S., G. A. Gagnon, D. R. Campbell and S. C. Clark (2011). Assessment of hydraulic flocculation processes using CFD. Journal American Water Works Association.

Versteeg, H. K. and W. Malalasekera (2007). An introduction to computational fluid dynamics: the finite volume method. Actas Urologicas Espanolas.

Vianna, M. R. (2019). Hydraulics Applied to Water Treatment Plant, 3i Editora Ltda, Belo Horizonte, Brazil.

Vianna, M. R., L. V. Castro and C. Oliveira (2008). Perforated tray-type hydraulic flocculator for potable water treatment: concept and state of the art in Brazil. International Journal of Emerging Technology and Advanced Engineering 5 (3), 5 7.

Wang, J., Y. Wu, H. Zhang and H. Jia (2014). Numerical and experimental investigation on integrated flocculation-membrane filtration process for the reactor configuration design and operational parameter optimization. Desalination.

Zhang, J., A. E. Tejada-Martínez and Q. Zhang (2014), Developments in computational fluid dynamics-based modeling for disinfection technologies over the last two decades: A review. Environmental Modelling and Software 